\title{
CTRP Intervention Terminology
}

National Cancer Institute

\section{Source}

National Cancer Institute. CTRP Intervention Terminology. NCI Thesaurus. Code

C118169.

A set of terminology that includes the interventions and therapies used in $\mathrm{NCl}$-sponsored clinical trials. 